Ann. Génét. Sél. anim., I975, 7 (2), I59-I66.

\title{
DÉTERMINISME GÉNÉTIQUE DU SYNDROME D'HYPERTHERMIE MALIGNE CHEZ LE PORC DE PIÉTRAIN
}

\author{
L. OLLIVIER, P. SELLIER et G. MONIN* \\ avec la collaboration technique de P. Dando, P. Vernin et A. TALmant \\ Station de Génétique quantitative et appliquée, \\ Centre national de Recherches zootechniques, I. N. R. A., \\ 78350 Jouy en Josas \\ * Station de Recherches sur la Viande, \\ Centre de Recherches de Clermont-Ferrand, I. N. R. A., \\ Theix, Saint Genès Champanelle, 63110 Beaumont
}

RÉSUMÉ

Un échantillon de 138 pores, de race de Piétrain, issus de 7 verrats et de 25 truies, a été soumis à une anesthésie à l'halothane, d'une durée maximum de 5 minutes; 39 porcs (soit 28 p.roo) ont manifesté le syndrome d'hyperthermie maligne. La fréquence des animaux manifestant le syndrome allait de o à $63 \mathrm{p}$. Ioo selon le père. Les données sont compatibles avec l'hypothèse d'un gène autosomal récessif à pénétrance élevée et de fréquence voisine de o,6. Le maintien de ce gène à des fréquences élevées dans certaines populations porcines pourrait s'expliquer par ses effets favorables sur le développement musculaire. Cette hypothèse s'appuie sur l'observation d'une musculature plus développée au sevrage chez les 5 verrats "porteurs " du gène que sur les 2 " non porteurs". Comme par ailleurs, chez le Piétrain, l'existence d'un gène majeur responsable de l'hypertrophie musculaire est soupçonnée, on peut penser qu'il s'agit d'un seul ct même gène. Cette hypothèse est encore incomplètement prouvée.

\section{INTRODUCTION}

Le syndrome d'hyperthermie maligne déclenché par l'anesthésie à l'halothane chez le Porc (HALL et al., I966) a donné lieu ces dernières années à de nombreuses études. Rappelons que ce syndrome est caractérisé par une élévation rapide de la température corporelle consécutive à des troubles du métabolisme musculaire. Les signes extérieurs les plus visibles sont la rigidité musculaire et 1'apparition de taches 
violacées sur la peau. La mort, qui s'ensuit normalement, peut être évitée si l'anesthésie est arrêtée dès les premières manifestations du syndrome. Par ailleurs, des réactions identiques, connues sous le nom de syndrome de stress, sont obtenues sur des porcs soumis à des " agressions " de nature diverse, telles que la chaleur, l'exercice physique violent, le transport à l'abattoir etc. Ces deux syndromes, hyperthermie maligne et stress, apparemment dus à un même désordre métabolique (BERMAN et $\mathrm{KENCH}, \mathrm{I97I}$ ), sont à l'origine de mortalités observées dans les conditions normales d'élevage et sont associés par ailleurs au phénomène de myopathie exsudative et dépigmentaire des viandes.

La base génétique de ces syndromes paraît établie (CHRISTIAN, I972), mais les opinions diffèrent quant au déterminisme exact : degré de dominance, pénétrance, fréquence de l'anomalie, etc. Nous nous proposons ici d'analyser le déterminisme génétique de l'anomalie en adaptant des méthodes proposées par LAUVERGNE et LEFORT (1973) et LAUVERGNE (I974)

\section{MATÉRIEL E'T MÉTHODES}

\section{I. - Matériel}

Un échantillon de 138 porcs de Piétrain ( 73 mâles, 65 femelles) a été soumis, de juillet à octobre 1974, à une épreuve d'anesthésie par halothane selon la technique décrite par ErkelenBOом et MiNkEMA (I974) : oxygène pur + halothane d̀ 2,5 p. Ioo, durée maximum 5 minutes. Ces porcs, âgés de 3 mois environ au moment de l'épreuve, étaient les produits d'unions au hasard dans une lignée entretenue au domaine expérimental I. N. R. A. d'Avord (Cher) à partir de reproducteurs achetés en 1968 dans les élevages de sélection français. Les 7 pères avaient subi, vers l'âge de 3 mois, un examen visuel de conformation aboutissant à une note (de o à I 2 ) attribuée selon le principe décrit par Ollivier et LAUVERGNe (1967). Cette note est proportionnelle à l'importance du développement musculaire tel qu'il peut être apprécié visuellement à cet âge.

\section{2. - Méthodes}

L'incidence du syndrome n'est pas connue chez les parents mais seulement chez les descendants qui se répartissent dans des familles de père constituées de germains et de demi-germains. L.a méthode consiste à : a) montrer que la tare est héréditaire, b) évaluer, dans l'hypothèse d'un déterminisme monofactoriel, autosomal, récessif, la pénétrance et la fréquence du gène.

\section{a) Mise en évidence de l'étiologie héréditaire du phénomène.}

Quand une tare n'est pas de nature héréditaire, il y a indépendance entre sa fréquence et le facteur père. La distribution du nombre d'anormaux par père suit alors une distribution binomiale (ou poissonnienne si la fréquence est faible) dans des familles de taille égale (LAUVERGNE, 1974). Nous avons soumis l'hypothèse d'une cause exogène, non liée au père, au test de $\chi^{2}$ de BRANDT et SNEDEcor, décrit par Kempthorne (r957).

\section{b) Fréquence et pénétrance du gène.}

Si maintenant nous faisons l'hypothèse la plus simple d'une tare monofactorielle, autosomale et récessive à pénétrance quelconque, il est possible d'appliquer la méthode décrite par LAUVERGNE et LEFORT (1973) qui consiste à estimer simultanément, à partir d'un échantillon de $l$ pères soumis à une épreuve de descendance, la fréquence $q(p=\mathbf{I}-q)$ du gène et sa pénétrance $w$, connaissant le nombre de descendants tarés de chaque père. Toutes les hypothèses décrites par ces auteurs ont été faites, à l'exception de celle concernant l'élimination des animaux tarés. Nous supposons donc que les 7 pères et les 25 mères de notre échantillon, dont le génotype est 
inconnu, n'ont donné lieu à aucune sélection entre la naissance et la mise à la reproduction. Dans ces conditions les probabilités des 3 génotypes chez les parents sont $p^{2}, 2 p q$ et $q^{2}$. Si nous désignons par :

$s_{0}$ le nombre de verrats reconnus transmetteurs de l'anomalie $\left(s_{0}=5\right)$,

$d_{0}$ le nombre de truies reconnues transmetteuses de l'anomalie $\left(d_{0}=16\right)$,

$m_{i}$ le nombre de portées du $i$ e verrat $(i=1, \ldots 7)$,

$n_{i j}$ l'effectif de la portée du $i$ e verrat et de la $j$ e truie,

$n_{0}$ l'effectif total des descendants $\left(n_{0}=\Sigma_{i j} n_{i j}=138\right)$,

$x_{0}$ le nombre total de descendants sensibles $\left(x_{0}=39\right)$,

l'espérance de la variable aléatoire $X_{0}$, nombre total de tarés observés, est :

$$
\mathrm{E}\left(\mathrm{X}_{0}\right)=n_{0}\left[q^{2}(q w)+2 p q(q w / 2)\right]=n_{0} q^{2} w
$$

Par ailleurs les probabilités qu'un descendant soit normal sont respectivement (1 - $w$ ), $(\mathrm{I}-w / 2)$ et $(\mathrm{I}-w / 4)$ selon que les 2 parents sont homozygotes récessifs, l'un homozygote récessif et l'autre hétérozygote, ou les deux hétérozygotes. D'où les probabilités qu'un père $i$ n'ait aucun anormal dans sa descendance sont respectivement :

et

$$
h_{i}=\prod_{j=1}^{m_{i}}\left\{p^{2}+2 p q(\mathrm{I}-w / 4)^{n_{i j}}+q^{2}(\mathrm{I}-w / 2)^{n_{i j}}\right\}
$$

$$
r_{i}=\prod_{j=1}^{m_{i}}\left\{p^{2}+2 p q(\mathrm{I}--w / 2)^{n_{i j}}+q^{2}(\mathrm{I}-w)^{n_{i j}}\right\}
$$

selon qu'il est homozygote normal, hétérozygote ou homozygote récessif. La probabilité qu'un père $i$ soit reconnu transmetteur de l'anomalie est donc :

$$
s_{i}=\mathbf{I}-\left(p^{2}+2 p q h_{i}+q^{2} r_{i}\right)
$$

Enfin la probabilité pour qu'une portée de $n_{i j}$ individus contienne au moins un anormal est :

$$
d_{i j}=q^{4}\left[\mathrm{I}-(\mathrm{I}-w)^{n_{i j}}\right]+4 p q^{3}\left[\mathrm{I}-(\mathrm{I}-w / 2)^{n_{i i}}\right]+4 p^{2} q^{2}\left[\mathrm{I}-(\mathrm{I}-w / 4)^{n_{i j}}\right]
$$

Les espérances des aléatoires $S_{0}$, nombre de pères transmetteurs, et $D_{0}$, nombre de mères transmetteuses, sont donc respectivement :

et

$$
\mathrm{E}\left(\mathrm{S}_{0}\right)=\sum_{i} s_{i}
$$

$$
\mathrm{E}\left(\mathrm{D}_{0}\right)=\sum_{i j} d_{i j}
$$

Les équations d'estimation sont obtenues par la méthode des moments en faisant $x_{0}=\mathrm{E}\left(\mathrm{X}_{0}\right), d_{0}=\mathrm{E}\left(\mathrm{D}_{0}\right), s_{0}=\mathrm{E}\left(\mathrm{S}_{0}\right)$ et cn cumulant les 2 dernières équations, ce qui donne le système :

$$
\text { (I) }\left\{\begin{array}{l}
x_{0}-n_{0} q^{2} w=0 \\
d_{0}+s_{0}-\sum_{i j} d_{i j}-\sum_{i} s_{i}=0
\end{array}\right.
$$

Le système ( $\mathrm{r}$ ) peut être résolu par itérations. Le détail de la méthode ainsi que l'obtention des variances des estimées est décrit par ailleurs (LEFORT et al., 1975).

\section{RÉSULTATS}

Le tableau I donne la note de conformation des 7 pères étudiés et la répartition des individus manifestant le syndrome dans les 7 descendances. La fréquence est très voisine dans les 2 sexes : $2 \mathrm{I}$ mâles et $\mathrm{r} 8$ femelles ont été trouvés sensibles. 
TABLEAU I

\begin{tabular}{|c|c|c|c|c|}
\hline \multirow{2}{*}{ Père } & \multirow{2}{*}{$\begin{array}{l}\text { Note de } \\
\text { conformation } \\
\text { du père }\end{array}$} & \multicolumn{2}{|c|}{ Portées examinées } & \multirow{2}{*}{$\begin{array}{c}\text { Fréquence } \\
\text { par père } \\
(\%)\end{array}$} \\
\hline & & Effectif & $\begin{array}{c}\text { Nombre de } \\
\text { sensibles }\end{array}$ & \\
\hline I & 1 & $\begin{array}{l}7 \\
7 \\
5 \\
4 \\
7\end{array}$ & $\begin{array}{l}0 \\
0 \\
0 \\
0 \\
0\end{array}$ & 0 \\
\hline II & 2 & 8 & 0 & 0 \\
\hline III & 4 & $\begin{array}{l}9 \\
2 \\
9 \\
4\end{array}$ & $\begin{array}{l}4 \\
2 \\
5 \\
4\end{array}$ & 63 \\
\hline IV & 5 & $\begin{array}{l}9 \\
4 \\
7\end{array}$ & $\begin{array}{l}1 \\
1 \\
3\end{array}$ & 25 \\
\hline $\mathrm{v}$ & 6 & $\begin{array}{l}1 \\
4\end{array}$ & $\begin{array}{l}0 \\
1\end{array}$ & 20 \\
\hline VI & 6 & $\begin{array}{r}7 \\
3 \\
10 \\
6 \\
2 \\
4\end{array}$ & $\begin{array}{l}1 \\
3 \\
0 \\
1 \\
1 \\
1\end{array}$ & 22 \\
\hline VII & 8 & $\begin{array}{l}7 \\
4 \\
4 \\
1\end{array}$ & $\begin{array}{l}6 \\
0 \\
2 \\
3\end{array}$ & 58 \\
\hline \multicolumn{2}{|c|}{ Total } & 138 & 39 & 28 \\
\hline
\end{tabular}

I. - Mise en évidence de la nature héréditaire du syndrome

La variabilité des fréquences de l'anomalie dans les 7 descendances du tableau I indique clairement l'existence d'un facteur génétique paternel, ce que confirme le test statistique de l'hypothèse d'indépendance : $\chi_{3}^{2}=39, \mathrm{P}<0,001$. L'anomalie ne peut donc être attribuée à une cause exogène.

\section{2. - Estimation des paramètres}

La base héréditaire du syndrome étant établie, la fréquence et la pénétrance du gène récessif postulé ont été estimées selon la méthode décrite plus haut. Les estimations obtenues (suivies de leur écart-type) sont : 


$$
\begin{aligned}
\widehat{q} & =0,64 \pm 0, \mathrm{r} 3 \\
\widehat{w} & =0,69 \pm 0,25
\end{aligned}
$$

L'hypothèse simple d'un gène autosomal récessif à pénétrance élevée et de fréquence moyenne est donc parfaitement compatible avec les données de cette expérience, comme cela apparaît d'ailleurs à l'examen des fréquences de l'anomalie par verrat : 2 verrats (probablement homozygotes dominants) donnant des fréquences nulles, 3 verrats (probablement hétérozygotes) donnant des fréquences voisines de $\mathrm{I} / 4$ et 2 verrats (probablement homozygotes récessifs) donnant des fréquences voisines de $x / 2$.

\section{DISCUSSION}

L'hypothèse d'une base génétique du syndrome d'hyperthermie maligne chez le Porc a d'abord été proposée par HaLI et al., (1966) qui, observant l'anomalie sur 3 pleins-frères, ont suggéré qu'il s'agirait d'un gène à forte pénétrance, sans toutefois émettre d'hypothèse quant au degré de dominance. CHristran (I972), après une revue détaillée et sur la base d'observations personnelles, conclut à un mutant récessif autosomal à pénétrance incomplète. Nos observations renforcent l'hypothèse de Christian sur la récessivité et ne s'en écartent guère quant à la pénétrance. Les différences minimes que l'on note peuvent s'expliquer à la fois par la taille limitée des échantillons observés et par l'absence d'estimations statistiques précises chez les précédents auteurs.

Remarquons que ces estimations de la pénétrance dépendent en premier lieu de la détection des réagissants et que la précision de celle-ci peut varier avec les modalités de l'anesthésie, principalement sa durée. En ce qui nous concerne, il semble cependant que peu de réagissants nous aient échappé, comme l'expliquerait d'ailleurs la valeur élevée de la pénétrance que nous estimons. En effet, même s'il n'est pas exclu que le syndrome puisse se déclencher au-delà des 5 minutes retenues lors de notre expérience, le temps moyen de réaction des 39 porcs positifs était néanmoins de l'ordre de 2 minutes et il semble établi par ailleurs que les premières manifestations $\mathrm{du}$ syndrome, en particulier le changement de concentration des électrolytes du sang et la rigidité musculaire qui lui est associée, sont très précoces et qu'il s'agit d'un phénomène de " tout ou rien " très répétable (BERMAN et al., I970; BERMAN et KENCH, I971).

Parmi les hypothèses sur lesquelles reposent les estimations, celle de l'absence de sélection chez les parents mérite d'être discutée. La sélection naturelle contre l'anomalie, dont les causes sont expliquées dans l'introduction, est probablement de faible intensité mais son existence nous conduit à sous-estimer la fréquence réelle du gène par la méthode ici employée. En contrepartie, comme nous allons le voir ci-dessous, la possibilité d'une sélection artificielle en faveur du gène ne doit pas être exclue.

Si on admet que notre échantillon est représentatif de la race - ce qui n'est pas rigoureusement établi puisqu'il s'agit seulement d'une lignée qui en dérive l'anomalie paraît être nettement plus fréquente chez le porc de Piétrain que chez le Landrace hollandais où la fréquence est de l'ordre de I3 p. IOO (EIKELENBoom et 
MrNkEma, I974). Il s'agit quand même, dans les deux cas, de fréquences assez élevées et on peut se demander comment ce gène, que la sélection naturelle tend à éliminer, se maintient malgré tout à de telles fréquences dans certaines populations porcines. L'explication pourrait résider dans une sélection artificielle s'exerçant à l'opposé de la sélection naturelle. Cette hypothèse implique, dans notre cas : (I) un effet pléiotropique du gène, (2) une sélection sur cet effet par les éleveurs, (3) des valeurs sélectives globales des 3 génotypes telles que l'on aboutisse à un équilibre génétique stable. Or :

- Un effet pléiotropique du gène sur le développement musculaire est suggéré par l'examen du tableau $I$, qui montre que les 5 verrats " porteurs du gène " (III à VII) avaient, vers l'âge de 3 mois, un développement musculaire apparent plus important que les 2 verrats "non porteurs " (I et II).

- On sait aussi, par ailleurs, qu'un fort développement musculaire a été recherché par les éleveurs de porcs de Piétrain, Landrace belge et leurs dérivés.

- Enfin, si on admet que la sélection des éleveurs s'exerce à l'encontre des homozygotes normaux - ce que suggère le tableau I - ou même si cette sélection affecte aussi les hétérozygotes, mais à un moindre degré, la sélection naturelle et la sélection artificielle se combinent pour aboutir à un avantage sélectif global des hétérozygotes pouvant expliquer le maintien du polymorphisme. Une telle situation ne serait pas entièrement nouvelle dans la pratique de l'élevage. A titre d'exemple très semblable, on pourrait citer le mécanisme qui, selon LAUVERGNE (I970), expliquerait le maintien dans la race bovine Suédoise des Montagnes d'un gène qui se comporte comme un récessif dans la détermination de 1'hypoplasie gonadique et comme un dominant quant à la coloration blanche du pelage, recherchée par les éleveurs.

Il est intéressant de rapprocher les observations qui viennent d'être faites, sur 1'aspect génétique de la sensibilité à 1'halothane et ses relations avec le développement musculaire, de l'existence supposée d'un gène majeur responsable de l'hypertrophie musculaire chez le porc de Piétrain (OLLIVIER et LAUVERGNE, I967; OlifiviER, I968). L'hypothèse qu'il s'agit d'un seul et même gène est tentante, aussi bien du point de vue théorique (" marquage " d'un gène de développement musculaire par une réaction physiologique bien caractérisée) que pratique (possibilité de détecter et d'utiliser les homozygotes), mais reste encore à prouver définitivement. D'autres cas de gènes majeurs à " effets pléiotropiques ambigus sur la productivité " sont également donnés par LAUVERGNE (I972).

\section{CONCLUSION}

Ces résultats, qui confirment la base génétique du syndrome d'hyperthermie maligne, ne constituent cependant pas une démonstration absolument rigoureuse du déterminisme génétique exact de l'anomalie. Ils montrent seulement qu'une hypothèse génétique simple est compatible avec ce que nous observons. Il reste à confirmer cette présomption par une étude détaillée d'accouplements entre animaux de phénotype déterminé. Par ailleurs, l'étude des relations entre ce syndrome et la 
composition corporelle ainsi que la qualité technologique des viandes est en cours. I a nature de ces relations conditionnera l'efficacité de la prise en considération de ce critère dans les programmes de sélection (OLLIVIER et POTIER, I975).

Reçu pour publication en mai 1975.

\section{REMERCIEMENTS}

Nous remercions le Dr J.-J. Lauvergne et le Pr G. Lefort pour leurs précieux conseils dans la rédaction de cet article ainsi que le Dr G. Eikelenboom pour la démonstration de sa technique avant la publication de ses propres résultats.

\section{SUMMARY}

GENETIC DETERMINATION

OF THE MALIGNANT HYPERTHERMIA SYNDROME IN THE PIETRAIN PIG

A sample of 138 Piétrain pigs, born from 7 boars and 25 sows, has been submitted to a 5-minute anesthesia with halothane ; 39 pigs (28 p. I0o) were sensitive and exhibited the malignant hyperthermia syndrome. The frequency of the syndrome went from o to $63 \mathrm{p}$. Ioo according to the boar progeny considered. The data agree with the hypothesis of a recessive gene, with high penetrance, and with a frequency near o.6. Such a gene might be maintained at a high frequency in some pig populations because of its favourable effects on muscular development. This hypothesis is suggested by the heavier muscling observed at weaning on the 5 "carrier " boars as compared to the 2 "non-carrier " ones. As also, in the Piétrain pig, one major gene is thought to be involved in muscular hypertrophy, one may hypothesize that the same one gene is responsible for both muscular hypertrophy and malignant hyperthermia. This however remains to be definitely proved.

\section{RÉFÉRENCES BIBI,IOGRAPHIQUES}

Berman M. C., Harrison G. G., Bull A. B., Kench J. E., r970. Changes underlying halothane induced malignant hyperpyrexia in Landrace pigs. Nature, 225, 653-655.

Berman M. C., Kench J. E., r971. Muscle metabolism during anaesthetic induced hyperthermia in pigs. Proc. 2nd int. Symp. Condition Meat Quality Pigs, 29-35, Pudoc, Wageningen.

Christian L. L., I972. A review of the role of genetics in animal stress susceptibility and meat quality. In, Cassens R., Giesler F., Kolb Q., Proc. Pork. Qual. Symp., 91-II5, Univ. Wisconsin, Madison.

Eikelenboom G., Minkema D., 1974. Prediction of pale, soft, exudative muscle with a non-lethal test for the halothane - induced porcine malignant hyperthermia syndrome. Neth. J. Vet. Sci., 99, $42 \mathrm{I}-426$.

Hall L. W., Woolf N., Bradley J. W. P., Jolly D. W., rg66. Unusual reaction to suxamethonium chloride. Brit. Med. J., 1966, 2, I305.

Kempthorne O., 1957. An Introduction to Genetic Statistics. John Wiley and Sons, New York, I5II54 et I74-I77.

Lauvergne J. J., 1970. Gonadal hypoplasia and white coat color in Swedish Highland cattle. J. Hered., 61, 43-44.

LAUVERGNe J. J., I972. Utilisation consciente et inconsciente de certains facteurs mendéliens daus la sélection des grosses espèces domestiques. Ann. Génét. Sél. anim., 4, 79-85.

LAUvergne J. J., 1974. Étude des gènes à effets visibles à partir des données de testage des taureaux sur leur descendance. 1er Congr. Mond. de Génét. appl. élev., 1, 47-55. 
Lauvergne J. J., Lefort G., 1973. Nouvelle méthode pour analyser le comportement et la fréquence des gènes récessifs à effets visibles dans les populations bovines. C. R. Acad. Sci. Paris, série D, 277, 2793-2796.

Lefort G., Ollivier L., Sellier P., 1975. Analyse du comportement et de la fréquence des gènes à effets visibles dans des fratries de germains et de demi-germains. Ann. Génet. Sel. anim. (sous presse).

Ollivier L., I968. Étude du déterminisme héréditaire de l'hypertrophie musculaire du porc de $P i E$ train. Ann. Zootech., 17, 393-407.

Olzivier L., Lauvergne J. J., r967. Étude du déterminisme héréditaire de l'hypertrophie musculaire du porc de Piđtrain : premiers résultats. Anrl. Méd. Vé., 111, 104-1o9.

Ollivier L., Potier D., I975. L'amélioration de la qualité de la viande porcine par la sélection. In Journés Rech. Porcine en France 1975, 293-302, Institut technique du Porc, Paris. 